\title{
The exact solution of the average run length on a modified EWMA control chart for the first-order moving-average process
}

\author{
Yadpirun Supharakonsakun, Yupaporn Areepong*, Saowanit Sukparungsee \\ Department of Applied Statistics, Faculty of Applied Science, King Mongkut's University of Technology \\ North Bangkok, Bangkok10800 Thailand
}

*Corresponding author, e-mail: yupaporn.a@sci.kmutnb.ac.th

Received 5 Sep 2019

Accepted 18 Feb 2020

\begin{abstract}
Statistical process control methods are widely used in several fields for monitoring and detecting process problems. One of them is the control chart which is powerful and effective for monitoring many types of processes, and its capability is usually measured using the average run length (ARL). In this article, we investigate explicit formulas for both the one- and the two-sided ARL on a modified exponentially weighted moving-average (EWMA) control chart for a first-order moving-average process with exponential white noise. The accuracy of the solution obtained with the modified EWMA control chart was compared to the numerical integral equation method and extended to a comparative performance with the standard EWMA control chart. The results show that the ARL obtained by the explicit formulas and the numerical integral equation method are in close agreement. The performance comparison shows that the modified EWMA control chart is dramatically more sensitive than the standard EWMA control chart for almost all of the studied exponential smoothing parameters and magnitudes of shift size. To demonstrate its capability, the proposed approach was applied to Thailand/US monthly foreign exchange rates data, yielding in good performance.
\end{abstract}

KEYWORDS: autocorrelated data, explicit formulas, monitoring process, statistical process control

MSC2010: 62P30 62P05 46N20

\section{INTRODUCTION}

Currently, the quality control of products or services, which plays an important role in business, industry, and manufacturing, can help in determining the characteristics of a product corresponding to customer demand. Moreover, products or services should have consistently maintained quality and standards. One of the most powerful sets of problem-solving tools for achieving process stability and improving capability through the reduction of variability is collectively called statistical process control (SPC) [1]. There are quite a few tools available for monitoring processes and detecting process deviation, and a most popular one is the control chart.

The first control chart widely used for monitoring processes and detecting out-of-control occurrences is the Shewhart control chart. It is effective for detecting large changes in the process mean and variance, but its performance will be degraded when small changes are detected, or the distribution of the assumption is not freeform. Many researchers have later proposed various control charts that are effective in detecting small changes, such as the cumulative sum (CUSUM) [2] and the exponentially weighted moving-average (EWMA) [3]. These control charts are well known for detecting SPC problems. More detection methods for small process shifts have been suggested by Yashchin [4], Zhang [5], Psarakis and Papaleonida [6], Prajapati [7], and Mawonike and Nkomo [8]. They have shown that the CUSUM and EWMA control charts are more effective than the Shewhart control chart for monitoring small changes and for autocorrelated processes. Recently, Patel and Divecha [9] proposed the modified EWMA control chart, developed from the original one, which is effective in detecting small and abrupt shifts in the mean of the monitored process. One of the benefits of using this control chart is that it performs well with observations that are serially correlated. Afterwards, Khan et al [10] proposed a new generalized structure for the modified EWMA statistic. The performance of the proposed control chart was compared with the original EWMA 
and modified EWMA control charts, as determined by their ARLs. The results indicate that the proposed control chart performed better than the two existing ones with each exponential smoothing parameter value.

One of the comparative performance methods for control charts is the average run length (ARL), which can be classified into two categories: $\mathrm{ARL}_{0}$ and $\mathrm{ARL}_{1}$, indicating the average number of points or observations plotted within the control limit until there exists a point or observation falsely identified as outside of the control limit. $\mathrm{ARL}_{0}$ is accepted if it is large enough to maintain false alarms at an acceptable level and thus it should be large. Meanwhile, $\mathrm{ARL}_{1}$ is the average number of points or observations plotted within the control limit from the change point time until there is a point or observation falsely identified outside of the control limit. $\mathrm{ARL}_{0}$ should be large whereas $\mathrm{ARL}_{1}$ should be as small as possible.

Many methods have been used to solve the exact solution of the ARL of a control chart, such as explicit formulas, along with several approaches to estimate the ARL, such as the Markov chain, Monte Carlo simulation, Martingale, and numerical integral equation (NIE).

Previous literature has focused on approximation of the ARL to represent an efficient control chart using many methods. Mastrangelo and Montgomery [11] evaluated the ARL of the traditional EWMA chart for serially correlated processes by using the Monte Carlo simulation method. Vanbrackle and Reynold [12] investigated EWMA and CUSUM control charts using the NIE and Markov chain approaches to find the ARL for a first-order autoregressive (AR(1)) process with additional random error. Fu et al [13] determined the run length and the ARL on CUSUM, EWMA and Shewhart control charts based on the Markov chain approach. Zhang et al [14] studied the efficiency of the EWMA control chart to further enhance the monitoring of the coefficient of variation when the process mean and standard deviation were not constant to determine the ARL, which was evaluated using Monte Carlo simulation approach.

In addition, there have been several researchers who computed the ARL by deriving explicit formulas and then comparing the ARL results with other methods to check the accuracy. Sukparungsee and Areepong [15] implemented an explicit formula for the ARL on a EWMA control chart, and compared the accuracy of the numerical results via Monte Carlo simulations. Busaba et al [16] analyzed explicit formulas for ARL on a CUSUM control chart for the case of a stationary AR(1) process and compared them with the NIE method. Meanwhile, Suriyakat et al [17] used the NIE method to solve the ARL and derived an explicit expression for AR(1) procedure on the EWMA control chart process. Areepong [18] proposed the explicit formulas on the moving-average (MA) control chart when observations are binomially distributed. Additionally, the new formulas were compared with the numerical simulation results of the Shewhart and EWMA charts. After that, Petcharat et al [19] derived explicit formulas for the ARL on an EWMA control chart for an MA process. Petcharat et al [20] analyzed explicit formulas and used the NIE method for the ARL on a CUSUM control chart when the observations comprised an MA of order $q(\mathrm{MA}(q))$. Phantu et al [21] proposed explicit formulas to evaluate the ARL of an MA control chart for an AR(1) serially dependent Poisson process. Peerajit et al [22] compared the efficiency of explicit solutions to the NIE method of ARL on a CUSUM control chart for a long memory process with a seasonally adjusted autoregressive fractionally integrated moving-average (ARFIMA) model. Later, Suntornwat et al [23] proposed an analytical solution for the ARL on EWMA control chart for an ARFIMA process by comparing it with the integral equation technique. Their findings show that the proposed methods of evaluation were in good agreement, but the explicit formulas had a faster computational time than the numerical ARL.

The performance of the EWMA and CUSUM control charts has been compared by a number of researchers. Vargas et al [24], Areepong[25], Suriyakat et al [26] and Phanyaem et al [27] which were determined by the ARL of the control charts. The results of these studies indicate that the EWMA control chart is more powerful than the others, and as previously mentioned, the modified EWMA control chart is more effective than the standard one for detecting the small shifts in the process and for autocorrelated data. The findings also indicate that the ARL is useful for comparing the efficiency of the control charts and that explicit formulas take much less computational time to evaluate the ARL than the other methods. Therefore, the derivation of explicit formulas on a modified EWMA chart is of interest in this study. The focus of this paper is on a comparison of explicit formulas with the NIE method by comparing their efficiencies on a modified EWMA control chart for a first-order movingaverage (MA(1)) process with exponential white noise. 


\section{MODIFIED EWMA CONTROL CHART}

The EWMA control chart continually monitors and detects small changes in the process mean. This was first proposed by Robert [3] in 1959. The EWMA control chart can be expressed by the recursive equation below.

$$
Z_{t}=(1-\lambda) Z_{t-1}+\lambda X_{t}, \quad t=1,2,3, \ldots,
$$

where $0<\lambda<1$ is an exponential smoothing parameter. The starting value is $Z_{0}=X_{0}$, the target value $\mu_{0}$, and $X_{t}$ is a processes with mean $\mu$ and variance $\sigma^{2}$. Then, the variance of $Z_{t}$ is $\sigma_{Z_{t}}^{2}=$ $\sigma^{2}\left(\frac{\lambda}{2-\lambda}\right)\left(1-(1-\lambda)^{2 i}\right)$. When $i$ gets large, the term $\left.(1-\lambda)^{2 i}\right)$ converges to 0 . Therefore, the general upper control limit (UCL) and lower control limit (LCL) to detect the sequence is given by,

$$
\begin{aligned}
& \mathrm{UCL}=\mu_{0}+L \sigma \sqrt{\frac{\lambda}{2-\lambda}}, \\
& \mathrm{LCL}=\mu_{0}-L \sigma \sqrt{\frac{\lambda}{2-\lambda}},
\end{aligned}
$$

where $\mu_{0}$ is the target mean, $\sigma$ is the process standard deviation, and $L$ is an appropriate control width limit.

A modified EWMA was developed and presented by Patel and Divecha [3] in 2011. It is a correction of the EWMA control statistic, and it is also free from the inertia problem. This is effective in detecting small and abrupt shifts when monitoring the process mean for observations that are independent and normally distributed or autocorrelated. Subsequently, the modified EWMA control statistic was redesigned by Khan et al [3] in 2017. They proposed the structure of control statistic which was developed of this chart to the more efficient with the traditional of EWMA and modified EWMA control charts.

The newly modified EWMA chart can be defined by the recursive equation below.

$$
Z_{t}=(1-\lambda) Z_{t-1}+\lambda X_{t}+c\left(X_{t}-X_{t-1}\right),
$$

where $c$ is a constant. The expected value and the variance of the modified EWMA control statistic are $E\left(Z_{0}\right)=\mu_{0}$ and $V\left(Z_{t}\right)=\left[\frac{\left(\lambda+2 \lambda c+2 c^{2}\right)}{2-\lambda}\right] \sigma^{2}$, respectively. Therefore, the upper control limit (UCL) and lower control limit (LCL) of the modified EWMA control chart are as follows,

$$
\begin{aligned}
& \mathrm{UCL}=\mu_{0}+Q \sigma \sqrt{\frac{\lambda+2 \lambda c+2 c^{2}}{2-\lambda}}, \\
& \mathrm{LCL}=\mu_{0}-Q \sigma \sqrt{\frac{\lambda+2 \lambda c+2 c^{2}}{2-\lambda}},
\end{aligned}
$$

where $\mu_{0}$ is the target mean, $\sigma$ is the process standard deviation, $Q$ is an appropriate control width limit, and $X_{t}$ is a sequence of observations. $Z_{0}=u$ and $X_{0}=v$ are the initial values and $0<\lambda \leqslant 1$ is an exponential smoothing parameter.

The stopping time of the EWMA chart is given by

$$
\tau_{h}=\inf \left\{t>0: Z_{t}<l \text { or } Z_{t}>h\right\},
$$

where $\tau_{k}$ is the stopping time, $h$ is the upper control limit (UCL) and $l$ is the lower control limit (LCL).

\section{ARL FOR MODIFIED EWMA CHART OF MA(1)}

Let MA(1) be observations of the first-order movingaverage process. This can be described by the following recursion.

$$
X_{t}=\mu+\epsilon_{t}-\theta \epsilon_{t-1},
$$

where $\epsilon_{t}$ is a white noise process assumed with the exponential distribution and $\theta$ is a moving-average coefficient with an initial value of $\epsilon_{0}=s$.

Therefore, the modified EWMA statistics $Z_{t}$ can be written as,

$$
\begin{aligned}
Z_{t}=(1-\lambda) Z_{t-1}- & X_{t-1}+(\lambda+c) \epsilon_{t} \\
& -(\lambda \theta+\theta c) \epsilon_{t-1}+(\lambda+c) \mu,
\end{aligned}
$$

where $0<\lambda \leqslant 1$ and the initial values $Z_{0}=u, X_{0}=$ $v, \epsilon_{0}=s, \mathrm{LCL}=a$, and $\mathrm{UCL}=b$. Thus,

$$
Z_{1}=(1-\lambda) u-v+(\lambda+c) \epsilon_{1}-(\lambda \theta+\theta c) s+(\lambda+c) \mu .
$$

The stopping time of the modified EWMA chart is given by

$$
\tau_{b}=\inf \left\{t>0: Z_{t}<a \text { or } Z_{t}>b\right\}
$$

where $\tau_{b}$ is the stopping time, $b$ is the upper control limit (UCL), and $a$ is the lower control limit (LCL).

Let $L(u)$ denote the average run length on the modified EWMA control chart. The integral equation can be written in the form

$$
L(u)=1+\int_{\frac{a-w}{\lambda+c}}^{\frac{b-w}{\lambda+c}} L((\lambda+c) y+w) f(y) \mathrm{d} y,
$$

where $w:=(1-\lambda) u-v-(\lambda \theta+\theta c) s+(\lambda+c) \mu$. Changing the variable, the integral equation becomes

$$
L(u)=1+\frac{1}{\lambda+c} \int_{a}^{b} L(k) f\left(\frac{k-w}{\lambda+c}\right) \mathrm{d} k .
$$


Therefore,

$$
\begin{aligned}
L(u)=1+\frac{1}{\lambda+c} \int_{a}^{b} L(k) \frac{1}{\beta} & \mathrm{e}^{\frac{-k+(1-\lambda) u-\nu-(\lambda \theta+\theta) s}{\beta(\lambda+c)}+\frac{\mu}{\beta}} \mathrm{d} k \\
& =1+\frac{C(u)}{\beta(\lambda+c)} D,
\end{aligned}
$$

where $C(u)=\mathrm{e}^{\frac{(1-\lambda) u-\nu-(\lambda \theta+\theta c) s}{\beta(\lambda+c)}+\frac{\mu}{\beta}}$ and

$$
\begin{aligned}
D & =\int_{a}^{b} L(k) \mathrm{e}^{-\frac{k}{\beta(\lambda+c)}} \mathrm{d} k \\
& =\int_{a}^{b}\left[1+\frac{C(k)}{\beta(\lambda+c)} D\right] \mathrm{e}^{-\frac{k}{\beta(\lambda+c)}} \mathrm{d} k \\
& =\int_{a}^{b} \mathrm{e}^{-\frac{k}{\beta(\lambda+c)}} \mathrm{d} k+\int_{a}^{b} \frac{\mathrm{e}^{\frac{(1-\lambda) k-\nu-(\lambda \theta+\theta c) s}{\beta(\lambda+c)}+\frac{\mu}{\beta}}}{\beta(\lambda+c)} \mathrm{e}^{-\frac{k}{\beta(\lambda+c)} D} \mathrm{~d} k \\
& =\frac{-\beta(\lambda+c)\left[\mathrm{e}^{-\frac{b}{\beta(\lambda+c)}}-\mathrm{e}^{-\frac{a}{\beta(\lambda+c)}}\right]}{1+\frac{1}{\lambda} \mathrm{e}^{-\frac{v}{\beta(\lambda+c)}-\frac{(\lambda \theta+\theta c) s}{\beta(\lambda+c)}+\frac{\mu}{\beta}}\left[\mathrm{e}^{-\frac{\lambda b}{\beta(\lambda+c)}}-\mathrm{e}^{-\frac{\lambda a}{\beta(\lambda+c)}}\right]} .
\end{aligned}
$$

Therefore,

$$
\begin{aligned}
L(u)= & 1+\frac{\mathrm{e}^{\frac{(1-\lambda) u-\nu-(\lambda \theta+\theta c) s}{\beta(\lambda+c)}+\frac{\mu}{\beta}}}{\beta(\lambda+c)} \\
& \times \frac{-\beta(\lambda+c)\left[\mathrm{e}^{-\frac{b}{\beta(\lambda+c)}}-\mathrm{e}^{\left.-\frac{a}{\beta(\lambda+c)}\right]}\right.}{1+\frac{1}{\lambda} \mathrm{e}^{-\frac{v-(\lambda \theta+\theta c) s}{\beta(\lambda+c)}+\frac{\mu}{\beta}}\left[\mathrm{e}^{-\frac{\lambda b}{\beta(\lambda+c)}}-\mathrm{e}^{-\frac{\lambda a}{\beta(\lambda+c)}}\right]} .
\end{aligned}
$$

Hence, the explicit two-sided formula of average run length for the first-order moving-average process on the modified EWMA control chart can be defined using the Fredholm integral equation of the second kind as follows,

$$
\mathrm{ARL}_{2-\text { sided }}=1-\frac{\lambda \mathrm{e}^{\frac{(1-\lambda) u}{\beta(\lambda+c)}}\left[\mathrm{e}^{-\frac{b}{\beta(\lambda+c)}}-\mathrm{e}^{-\frac{a}{\beta(\lambda+c)}}\right]}{\lambda \mathrm{e}^{\frac{-\mu}{\beta}+\frac{v+(\lambda \theta+\theta c) s}{\beta(\lambda+c)}}+\mathrm{e}^{-\frac{\lambda b}{\beta(\lambda+c)}}-\mathrm{e}^{-\frac{\lambda a}{\beta(\lambda+c)}} .}
$$

When $a=0$, the explicit one-sided formulas of average run length on the modified EWMA control chart can be defined as follows,

$$
\mathrm{ARL}_{1-\text { sided }}=1-\frac{\lambda \mathrm{e}^{\frac{(1-\lambda) u}{\beta(\lambda+c)}}\left[\mathrm{e}^{-\frac{b}{\beta(\lambda+c)}}-1\right]}{\lambda \mathrm{e}^{\frac{-\mu}{\beta}+\frac{v+(\lambda \theta+\theta c) s}{\beta(\lambda+c)}}+\mathrm{e}^{-\frac{\lambda b}{\beta(\lambda+c)}}-1},
$$

with in-control process parameter $\beta=\beta_{0}=1$ and out of control process parameter $\beta=\beta_{1}>1$.

\section{EXISTENCE AND UNIQUENESS OF ARL}

The solution of average run length shows that there uniquely exists the integral equation for explicit formulas by the Banach's Fixed-point Theorem. In this study, let $T$ be an operation in the class of all continuous functions defined by

$$
T(L(u))=1+\frac{1}{(\lambda+c)} \int_{a}^{b} L(k) \frac{1}{\beta} \mathrm{e}^{\frac{-k+(1-\lambda) u-v-(\lambda \theta+\theta c) s}{\beta(\lambda+c)}+\frac{\mu}{\beta}} \mathrm{d} k .
$$

According to Banach's Fixed-point Theorem, if an operator $T$ is a contraction, then the fixed-point equation $T(L(u))=L(u)$ has a unique solution. To show that (14) exists and has a unique solution, the following theorem can be used.

Theorem 1 (Banach Fixed-point) Let $(X, d)$ be a complete metric space and $T: X \rightarrow X$ be a contraction mapping with contraction constant $0 \leqslant r<1$ such that $\left\|T\left(L_{1}\right)-T\left(L_{2}\right)\right\| \leqslant r\left\|L_{1}-L_{2}\right\|$ for all $L_{1}, L_{2} \in$ $X$. Then there exists a unique $L(\cdot) \in X$ such that $T(L(u))=L(u)$, i.e., a unique fixed-point in $X$ [28].

Proof: To show that $T$ defined in (14) is a contraction mapping for $L_{1}, L_{2} \in C[a, b]$, we will show that $\left\|T\left(L_{1}\right)-T\left(L_{2}\right)\right\| \leqslant r\left\|L_{1}-L_{2}\right\|$ for all $L_{1}, L_{2} \in$ $C[a, b]$ with $0 \leqslant r<1$ under the norm $\|L\|_{\infty}=$ $\sup _{u \in(a, b)}|L(u)|$. From (11) and (14),

$$
\begin{aligned}
& \left\|T\left(L_{1}\right)-T\left(L_{2}\right)\right\|_{\infty} \\
& =\sup _{u \in(a, b)}\left|\frac{C(u)}{\beta(\lambda+c)} \int_{a}^{b}\left(L_{1}(k)-L_{2}(k)\right) \mathrm{e}^{-\frac{k}{\beta(\lambda+c)}} \mathrm{d} k\right| \\
& \leqslant \sup _{u \in(a, b)}\left|\left\|L_{1}-L_{2}\right\|_{\infty} C(u)\left(\mathrm{e}^{\frac{-a}{\beta(\lambda+c)}}-\mathrm{e}^{\frac{-b}{\beta(\lambda+c)}}\right)\right| \\
& =\left\|L_{1}-L_{2}\right\|_{\infty} \sup _{u \in(a, b)}|C(u)|\left|\mathrm{e}^{\frac{-a}{\beta(\lambda+c)}}-\mathrm{e}^{\frac{-b}{\beta(\lambda+c)}}\right| \\
& \leqslant r\left\|L_{1}-L_{2}\right\|_{\infty},
\end{aligned}
$$

where $r:=\sup _{u \in(a, b)}|C(u)|\left|\mathrm{e}^{\frac{-a}{\beta(\lambda+c)}}-\mathrm{e}^{\frac{-b}{\beta(\lambda+c)}}\right|$ and $C(u)=\mathrm{e}^{\frac{(1-\lambda) u-v-(\lambda \theta+\theta) s}{\beta(\lambda+c)}+\frac{\mu}{\beta}}=\mathrm{e}^{\frac{(1-\lambda) u}{\beta(\lambda+c)}-\frac{v}{\beta(\lambda+c)}-\frac{\theta s}{\beta}+\frac{\mu}{\beta}} ; 0 \leqslant r<1$.

Therefore, the existence and the uniqueness of the solution are guaranteed by the Banach's Fixedpoint Theorem.

\section{NUMERICAL INTEGRAL EQUATION METHOD}

According to the integral equation in (10), the numerical integral equation (NIE) method can be used to evaluate the solution using the Gauss-Legendre quadrature rule as follows.

$L\left(a_{i}\right)=1+\frac{1}{\lambda+c} \sum_{j=1}^{m} w_{j} L\left(a_{j}\right) f\left(\frac{a_{j}-(1-\lambda) a_{i}+v}{\lambda+c}+\theta s-\mu\right)$, 
where $i=1,2,3, \ldots, m, a_{j}=\frac{b-a}{m}\left(j-\frac{1}{2}\right)+a$, and $w_{j}=$ $\frac{b-a}{m}, j=1,2,3, \ldots, m$.

This can be rewritten in a matrix form as

$$
\begin{aligned}
L_{m \times 1} & =1_{m \times 1}+R_{m \times m} L_{m \times 1} \\
\text { or } \quad L_{m \times 1} & =\left(I_{m}-R_{m \times m}\right)^{-1} 1_{m \times 1},
\end{aligned}
$$

where $L_{m \times 1}=\left[\begin{array}{c}L\left(a_{1}\right) \\ \vdots \\ L\left(a_{m}\right)\end{array}\right], 1_{m \times 1}=\left[\begin{array}{c}1 \\ \vdots \\ 1\end{array}\right], I_{m}$ is the

identity, and $R_{m \times m}=\frac{1}{\lambda+c}\left[\begin{array}{ccc}w_{1} f_{11} & \cdots & w_{m} f_{1 m} \\ w_{1} f_{21} & \cdots & w_{m} f_{2 m} \\ \vdots & & \vdots \\ w_{1} f_{m 1} & \cdots & w_{m} f_{m m}\end{array}\right]$,

when $f_{i j}:=f\left(\frac{a_{j}-(1-\lambda) a_{i}+v}{\lambda+c}+\theta s-\mu\right)$.

Therefore, the approximation of average run length is evaluated by NIE method for $L(u)$ is

$$
\tilde{L}(u)=1+\frac{1}{\lambda+c} \sum_{j=1}^{m} w_{j} L\left(a_{j}\right) f\left(\frac{a_{j}-(1-\lambda) u+v}{\lambda+c}+\theta s-\mu\right) .
$$

\section{NUMERICAL RESULTS}

In this section, the ARL was approximated by NIE method using the Gauss-Legendre quadrature rule on a modified EWMA control chart with 1000 nodes. The accuracy of ARL was used to measure the absolute percentage relative error (APRE),

$$
\operatorname{APRE}(\%)=\frac{|L(u)-\tilde{L}(u)|}{L(u)} \times 100,
$$

where $\tilde{L}(u)$ is an approximation of ARL using NIE method. The numerical results are computed by MATHEMATICA. The evaluation of numerical results using (12) and (13) are shown in Table 1 and Table 2, respectively. The explicit one-sided formula of ARL and NIE method on modified EWMA control chart for the first-order moving-average when given $\mathrm{ARL}_{0}=370, c=50 \lambda, \lambda=0.05,0.1$, and $\theta=$ $-0.1,0.1,-0.5,0.5$ were in dramatic agreement. But, the computational time of the explicit formulas is not much, while the numerical integral equation method took around $22 \mathrm{~s}$ for the one-sided of ARL and $28-30 \mathrm{~s}$ for the two-sided of ARL.

\section{COMPARISON OF PERFORMANCE FOR EWMA AND MODIFIED EWMA CHARTS}

In this section, the numerical comparative results of ARL on the modified EWMA and EWMA control charts are investigated. The proposed expression of

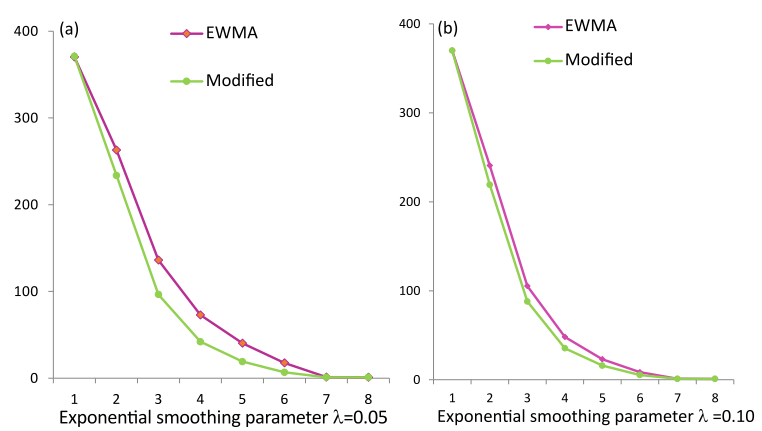

Fig. 1 The ARL of the EWMA and modified EWMA control charts with real data: (a) $\lambda=0.05$ and (b) $\lambda=0.10$.

modified EWMA chart was computed by (12) and (13). The explicit formulas of average run length in EWMA control chart for moving average order $q$ process with exponential white noise was applied to the first-order moving-average process, as proposed by Petcharat et al.

Table 4 and Table 5 present the ARL of explicit formulas on EWMA and modified EWMA control charts for the first-order moving average process when $\mathrm{ARL}_{0}=370,500, \mu=2, \lambda=0.05,0.1, \theta=$ $-0.2,0.2,-0.4,0.4$, which are obtained by the upper control limit and lower control limit (Table 3). The results indicated that modified EWMA immediately reduced the $\mathrm{ARL}_{1}$ more than the EWMA control chart when detecting the mean shift process. It can be said that the performance of modified EWMA control chart is more powerful than the performance of EWMA control chart for most scales of shift size.

\section{APPLICATION}

In this section, real data was applied to determine the average run length by the explicit formulas on modified EWMA and EWMA control charts for the Thailand/US foreign exchange rates observed from April 2014 to March 2019. This data is a stationary time series. By looking at the autocorrelation function (ACF) and partial autocorrelation (PACF). The data was analyzed and fitted with the firstorder of moving-average process with the significant of mean and coefficient 33.567 and -0.869 , respectively, and the white noise was significantly exponentially distributed with mean 0.8207 . The average run lengths on modified EWMA and EWMA control charts with the results of real data are shown in Table 6 and Fig. 1.

Table 6 shows that the results are in similar agreement to Table 4 and Table 5. The modified EWMA control chart offers an $\mathrm{ARL}_{1}$ that is more 
Table 1 The one-sided of ARL for MA(1) on the modified EWMA chart using explicit formula and NIE when $\mathrm{ARL}_{0}=370$, $\mu=2$, and $c=50 \lambda$.

\begin{tabular}{|c|c|c|c|c|c|c|c|}
\hline \multirow[t]{2}{*}{$\theta$} & \multirow{2}{*}{$\begin{array}{c}\text { Shift size } \\
\qquad(\delta)\end{array}$} & \multicolumn{3}{|c|}{$\lambda=0.05^{*}$} & \multicolumn{3}{|c|}{$\lambda=0.1^{* *}$} \\
\hline & & Explicit & NIE & APRE & Explicit & NIE & APRE \\
\hline \multirow[t]{8}{*}{-0.1} & 0.00 & 370.0000867370 & $\begin{array}{c}370.0000858815 \\
(21.918)\end{array}$ & $2.312 \times 10^{-7}$ & 370.0000606318 & $\begin{array}{c}370.0000591485 \\
(21.809)\end{array}$ & $4.009 \times 10^{-7}$ \\
\hline & 0.01 & 44.0439249625 & $\begin{array}{c}44.0439249000 \\
(21.980)\end{array}$ & $1.419 \times 10^{-7}$ & 35.5693493235 & $\begin{array}{c}35.5693492821 \\
(22.059)\end{array}$ & $1.164 \times 10^{-7}$ \\
\hline & 0.03 & 16.3214268274 & $\begin{array}{c}16.3214268070 \\
(21.949)\end{array}$ & $1.250 \times 10^{-7}$ & 13.1229621168 & $\begin{array}{c}13.1229621050 \\
(21.995)\end{array}$ & $8.992 \times 10^{-8}$ \\
\hline & 0.05 & 10.2080506715 & $\begin{array}{c}10.2080506598 \\
(22.012)\end{array}$ & $1.146 \times 10^{-7}$ & 8.2715123064 & $\begin{array}{c}8.2715122999 \\
(22.028)\end{array}$ & $7.858 \times 10^{-8}$ \\
\hline & 0.07 & 7.5274541620 & $\begin{array}{c}7.5274541541 \\
(22.012)\end{array}$ & $1.049 \times 10^{-7}$ & 6.1546487285 & $\begin{array}{c}6.1546487241 \\
(22.105)\end{array}$ & $7.149 \times 10^{-8}$ \\
\hline & 0.10 & 5.4963051443 & $\begin{array}{c}5.4963051391 \\
(22.106)\end{array}$ & $9.461 \times 10^{-8}$ & 4.5543136310 & $\begin{array}{c}4.5543136282 \\
(22.090)\end{array}$ & $6.148 \times 10^{-8}$ \\
\hline & 0.30 & 2.3371054262 & $\begin{array}{l}2.3371054251 \\
\quad(22.152)\end{array}$ & $4.707 \times 10^{-8}$ & 2.0669730690 & $\begin{array}{c}2.0669730685 \\
(21.996)\end{array}$ & $2.419 \times 10^{-8}$ \\
\hline & 0.50 & 1.7313103221 & $\begin{array}{c}1.7313103217 \\
(22.183)\end{array}$ & $4.707 \times 10^{-8}$ & 1.5875989978 & $\begin{array}{c}1.5875989976 \\
(22.074)\end{array}$ & $1.260 \times 10^{-8}$ \\
\hline \multirow[t]{8}{*}{0.1} & 0.00 & 370.0000191101 & $\begin{array}{c}370.0000178192 \\
(21.886)\end{array}$ & $3.489 \times 10^{-7}$ & 370.0000939896 & $\begin{array}{c}370.0000917457 \\
(21.784)\end{array}$ & $6.065 \times 10^{-7}$ \\
\hline & 0.01 & 46.3721160788 & $\begin{array}{c}46.3721159797 \\
(21.924)\end{array}$ & $2.137 \times 10^{-7}$ & 37.4509233915 & $\begin{array}{c}37.4509233251 \\
(21.895)\end{array}$ & $1.773 \times 10^{-7}$ \\
\hline & 0.03 & 17.2560948857 & $\begin{array}{c}17.2560948533 \\
(22.013)\end{array}$ & $1.878 \times 10^{-7}$ & 13.8543677226 & $\begin{array}{c}13.8543677037 \\
(21.893)\end{array}$ & $1.364 \times 10^{-7}$ \\
\hline & 0.05 & 10.7988295175 & $\begin{array}{c}10.7988294989 \\
(22.028)\end{array}$ & $1.722 \times 10^{-7}$ & 8.7312602017 & $\begin{array}{c}8.7312601912 \\
(21.784)\end{array}$ & $1.203 \times 10^{-7}$ \\
\hline & 0.07 & 7.9626558548 & $\begin{array}{c}7.9626558422 \\
(22.086)\end{array}$ & $1.582 \times 10^{-7}$ & 6.4928549093 & $\begin{array}{l}6.4928549023 \\
\quad(22.014)\end{array}$ & $1.078 \times 10^{-7}$ \\
\hline & 0.10 & 5.8110939946 & $\begin{array}{c}7.9626558422 \\
(22.098)\end{array}$ & $1.411 \times 10^{-7}$ & 4.7989996955 & $\begin{array}{l}4.7989996909 \\
(21.998)\end{array}$ & $9.585 \times 10^{-8}$ \\
\hline & 0.30 & 2.4548556274 & $\begin{array}{c}2.4548556256 \\
(22.174)\end{array}$ & $7.332 \times 10^{-8}$ & 2.1594952549 & $\begin{array}{c}2.1594952585 \\
(22.087)\end{array}$ & $1.667 \times 10^{-7}$ \\
\hline & 0.50 & 1.8060957035 & $\begin{array}{l}1.8060957028 \\
(22.192)\end{array}$ & $3.876 \times 10^{-8}$ & 1.6469191130 & $\begin{array}{c}1.6469191126 \\
(22.124)\end{array}$ & $2.429 \times 10^{-7}$ \\
\hline
\end{tabular}

\footnotetext{
" $b=0.4626313926$ for $\theta=-0.1$ and $b=0.565666747$ for $\theta=0.1$.

${ }^{* *} b=0.763204934$ for $\theta=-0.1$ and $b=0.933777249$ for $\theta=0.1$.
}

promptly deducted and very small for all shift sizes and exponential smoothing parameter values. This means that the performance of the modified EWMA chart was more powerful than the EWMA chart for all cases when monitoring and detecting the change of mean.

The exchange rate is an indicator that is measured in terms of national currency per US dollar. It is defined as the price of national currency in relation to the US dollar, and is expressed as the average rate for a period of time. In this case, there were 60 observations of monthly Thailand exchange rates of Thailand/US Dollar from April 2014 to March 2019 analyzed. The mean and standard deviation of the time series data were 33.5904 and 1.45699 , respectively. The upper and lower control limits were established by (2) and (3) for the modified EWMA control chart and (5) and (6) for the EWMA control chart. The detection of the process with real data is presented in Fig. 2. These show that the modified EWMA control chart is superior to the
EWMA control chart at the 5th observation when detecting changes, while the EWMA control chart can detect at the 25th observation for the out-ofcontrol process.

Fig. 2 indicates that the modified EWMA chart was able to detect the exchange rate at the 17th24th, the 26th, 27th, and 32th-34th observations. These observations were plotted above the upper control limit. One could suspect that the depreciation has occurred at or before that time. Thus, the depreciation of the currency is an important indicator in price determination. This situation directly affects the direct import business. On the other hand, if the observations are plotted below the lower control limit, it means appreciation will occur and will also impact the export business. Therefore, tracking changes in exchange rates is the key for high performance in both economic and financial areas. 
Table 2 The two-sided of ARL for MA(1) on the modified EWMA chart using explicit formula and NIE for ARL ${ }_{0}=370$, $\mu=2$, and $c=50 \lambda$.

\begin{tabular}{|c|c|c|c|c|c|c|c|}
\hline \multirow[t]{2}{*}{$\theta$} & \multirow{2}{*}{$\begin{array}{c}\text { Shift size } \\
(\delta)\end{array}$} & \multicolumn{3}{|c|}{$\lambda=0.05^{*}$} & \multicolumn{3}{|c|}{$\lambda=0.1^{* * *}$} \\
\hline & & Explicit & NIE & APRE & Explicit & NIE & APRE \\
\hline \multirow[t]{8}{*}{-0.5} & 0.00 & 370.0000694633 & $\begin{array}{c}370.0000687600 \\
(30.139)\end{array}$ & $1.901 \times 10^{-7}$ & 370.0000980167 & $\begin{array}{c}370.0000965317 \\
(28.533)\end{array}$ & $4.014 \times 10^{-7}$ \\
\hline & 0.01 & 41.5661928805 & $\begin{array}{c}41.5661928328 \\
(28.735)\end{array}$ & $1.148 \times 10^{-7}$ & 34.9758342828 & 34.9758342426 & $1.149 \times 10^{-7}$ \\
\hline & 0.03 & 15.3646262066 & $\begin{array}{c}15.3646261911 \\
(28.470)\end{array}$ & $1.009 \times 10^{-7}$ & 12.9043398195 & $\begin{array}{c}12.9043398080 \\
(30.311)\end{array}$ & $8.912 \times 10^{-8}$ \\
\hline & 0.05 & 9.6209444263 & $\begin{array}{c}9.6209444175 \\
(28.548)\end{array}$ & $9.147 \times 10^{-8}$ & 8.1405113753 & $\begin{array}{c}8.1405113690 \\
(28.298)\end{array}$ & $7.739 \times 10^{-8}$ \\
\hline & 0.07 & 7.1063784712 & $\begin{array}{c}7.1063784652 \\
(28.564)\end{array}$ & $8.443 \times 10^{-8}$ & 6.0625383282 & $\begin{array}{c}6.0625383240 \\
(29.032)\end{array}$ & $6.928 \times 10^{-8}$ \\
\hline & 0.10 & 5.2026737276 & $\begin{array}{c}5.2026737237 \\
(28.985)\end{array}$ & $7.496 \times 10^{-8}$ & 4.4917979139 & $\begin{array}{c}4.4917979111 \\
(28.611)\end{array}$ & $6.148 \times 10^{-8}$ \\
\hline & 0.30 & 2.2450744171 & $\begin{array}{c}2.2450744162 \\
(27.753)\end{array}$ & $4.009 \times 10^{-8}$ & 2.0501611023 & 2.0501611018 & $2.439 \times 10^{-8}$ \\
\hline & 0.50 & 1.6789252310 & $\begin{array}{c}1.6789252307 \\
(29.469)\end{array}$ & $1.787 \times 10^{-8}$ & 1.5791522674 & $\begin{array}{c}1.5791522672 \\
(27.738)\end{array}$ & $1.267 \times 10^{-8}$ \\
\hline \multirow[t]{8}{*}{0.5} & 0.00 & 370.0000411179 & $\begin{array}{c}370.0000394821 \\
(28.954)\end{array}$ & $4.421 \times 10^{-7}$ & 370.0000889780 & $\begin{array}{c}370.0000866536 \\
(29.078)\end{array}$ & $6.065 \times 10^{-7}$ \\
\hline & 0.01 & 46.2382379117 & $\begin{array}{c}46.2382377884 \\
(30.108)\end{array}$ & $2.667 \times 10^{-7}$ & 36.9785201623 & $\begin{array}{c}36.9785200953 \\
(28.705)\end{array}$ & $1.812 \times 10^{-7}$ \\
\hline & 0.03 & 17.2318873875 & $\begin{array}{c}17.2318873473 \\
(29.515)\end{array}$ & $1.878 \times 10^{-7}$ & 13.6814661485 & $\begin{array}{c}13.6814661295 \\
(28.891)\end{array}$ & $1.389 \times 10^{-7}$ \\
\hline & 0.05 & 10.8006895819 & $\begin{array}{c}10.8006895588 \\
(28.345)\end{array}$ & $2.139 \times 10^{-7}$ & 8.6289415891 & $\begin{array}{c}8.6289415785 \\
(29.235)\end{array}$ & $1.228 \times 10^{-7}$ \\
\hline & 0.07 & 7.9756157868 & $\begin{array}{c}7.9756157711 \\
(29.731)\end{array}$ & $1.968 \times 10^{-7}$ & 6.4218897912 & $\begin{array}{c}6.4218897842 \\
(28.376)\end{array}$ & $1.090 \times 10^{-7}$ \\
\hline & 0.10 & 5.8317979184 & $\begin{array}{c}5.8317979081 \\
(28.096)\end{array}$ & $1.766 \times 10^{-7}$ & 4.7518643793 & $\begin{array}{l}4.7518643748 \\
(30.701)\end{array}$ & $9.470 \times 10^{-8}$ \\
\hline & 0.30 & 2.4815717824 & $\begin{array}{c}2.4815717801 \\
(28.283)\end{array}$ & $9.268 \times 10^{-8}$ & 2.1487289737 & $\begin{array}{c}2.1487289727 \\
(29.032)\end{array}$ & $4.654 \times 10^{-8}$ \\
\hline & 0.50 & 1.8297312330 & $\begin{array}{c}1.8297312320 \\
(28.673)\end{array}$ & $5.465 \times 10^{-8}$ & 1.6424658227 & $\begin{array}{c}1.6424658223 \\
(29.297)\end{array}$ & $2.435 \times 10^{-8}$ \\
\hline
\end{tabular}

" $a=0.1, b=0.5168237377$ for $\theta=-0.5$ and $a=0.1, b=0.7305875693$ for $\theta=0.5$.

** $a=0.1, b=0.858734671$ for $\theta=-0.5$ and $a=0.1, b=1.043114241$ for $\theta=0.5$.

Table 3 Upper control limit and lower control limit of EWMA and modified EWMA charts using explicit formulas.

\begin{tabular}{|c|c|c|c|c|c|c|c|c|c|}
\hline \multirow{3}{*}{$\mathrm{ARL}_{0}$} & \multirow{3}{*}{$\theta$} & \multicolumn{4}{|c|}{$\lambda=0.05$} & \multicolumn{4}{|c|}{$\lambda=0.1$} \\
\hline & & \multicolumn{2}{|r|}{ EWMA } & \multicolumn{2}{|r|}{ Mofified } & \multicolumn{2}{|r|}{ EWMA } & \multicolumn{2}{|r|}{ Modified } \\
\hline & & $l$ & $h$ & $a$ & $b$ & $l$ & $h$ & $a$ & $b$ \\
\hline \multirow[t]{2}{*}{370} & -0.4 & 0.1 & 0.1000084203 & 0.1 & 0.534425014 & 0.1 & 0.5148186 & 0.1 & 0.875406997 \\
\hline & 0.4 & 0.1 & 0.100003783309 & 0.1 & 0.7049886465 & 0.1 & 0.17579211 & 0.1 & 1.0228024732 \\
\hline \multirow[t]{2}{*}{500} & -0.4 & 0.1 & 0.100011387083 & 0.1 & 0.5348217905 & 0.1 & 0.9169484 & 0.1 & 0.8760101704 \\
\hline & 0.4 & 0.1 & 0.1000051162256 & 0.1 & 0.7055255095 & 0.1 & 0.210902 & 0.1 & 1.2035123745 \\
\hline
\end{tabular}

\section{CONCLUSION}

Explicit formulas for the ARL on a modified EWMA control chart monitoring an MA(1) process with exponential white noise which provides its own expression that is easy to derive and saves on computation time was investigated in this research. The accuracy of the explicit formulas was checked by comparing its absolute percentage relative error with that of the NIE method. The results show that both methods were in dramatic agreement with absolute percentage errors of less than $9.212 \times$ $10^{-7}$. The exponential smoothing parameter in the range of $0.05-0.25$ is usually recommended. A comparison of the ARL performance of the modified and standard EWMA control charts shows that the former was more sensitive when shift sizes were small and performed better when the exponential smoothing parameter value was 0.1 . The exponential smoothing parameter 0.05 is unsuitable to the most effective of the modified EWMA chart employ- 
Table 4 Comparison of one-sided of ARL for MA(1) on EWMA and modified EWMA charts using explicit formulas when $\mu=2$ and $c=50 \lambda$.

\begin{tabular}{|c|c|c|c|c|c|c|}
\hline \multirow{2}{*}{$\lambda$} & \multirow[t]{2}{*}{$\theta$} & \multirow{2}{*}{$\begin{array}{c}\text { Shift size } \\
(\delta)\end{array}$} & \multicolumn{2}{|c|}{$\mathrm{ARL}_{0}=370$} & \multicolumn{2}{|c|}{$\mathrm{ARL}_{0}=500$} \\
\hline & & & EWMA & Modified & EWMA & Modified \\
\hline \multirow[t]{16}{*}{0.05} & \multirow[t]{8}{*}{-0.2} & 0.00 & 370.0000756855 & 370.0000433201 & 500.0000550446 & 500.0000517554 \\
\hline & & 0.01 & 310.3613470534 & 42.9524104373 & 419.3504170920 & 44.2714306321 \\
\hline & & 0.03 & 220.6242217364 & 15.8864511633 & 297.9986166729 & 16.0587690464 \\
\hline & & 0.05 & 158.9071572943 & 9.9337191786 & 214.5384272538 & 9.9990002172 \\
\hline & & 0.07 & 115.9013063561 & 7.3256434174 & 156.3814690365 & 7.3601034492 \\
\hline & & 0.10 & 73.8363408663 & 5.3505613736 & 99.4968683696 & 5.3681504949 \\
\hline & & 0.30 & 6.8797381907 & 2.2829992334 & 8.9511975571 & 2.2853882186 \\
\hline & & 0.50 & 1.9096782195 & 1.6971565736 & 2.2301626398 & 1.6981625206 \\
\hline & \multirow[t]{8}{*}{0.2} & 0.00 & 370.0000465698 & 370.0000749812 & 500.0000461456 & 500.0000637535 \\
\hline & & 0.01 & 309.1385402792 & 47.6155843164 & 417.6968310349 & 49.2416289066 \\
\hline & & 0.03 & 218.0802984841 & 17.7591290169 & 294.5584663025 & 17.9747029006 \\
\hline & & 0.05 & 155.9278248873 & 11.1174992983 & 210.5094640643 & 11.1993602969 \\
\hline & & 0.07 & 112.9335052931 & 8.1977303757 & 152.3680959291 & 8.2409666592 \\
\hline & & 0.10 & 71.2352935291 & 5.9813923738 & 95.9794555925 & 6.0034609210 \\
\hline & & 0.30 & 6.3612841228 & 2.5190268777 & 8.2500877220 & 2.5220254650 \\
\hline & & 0.50 & 1.7961249424 & 1.8470923312 & 2.0766034871 & 1.8383611231 \\
\hline \multirow[t]{16}{*}{0.10} & \multirow[t]{8}{*}{-0.2} & 0.00 & 370.0000618877 & 370.0000321922 & 500.0000893066 & 500.0000737559 \\
\hline & & 0.01 & 343.5592416319 & 34.6891029378 & 464.817836050466 & 35.5348763989 \\
\hline & & 0.03 & 297.4261061703 & 12.7828116715 & 403.3251666153 & 12.8906618547 \\
\hline & & 0.05 & 258.8319280712 & 8.0580837849 & 351.7620972277 & 8.0988617372 \\
\hline & & 0.07 & 226.3579081554 & 5.9978247468 & 308.2779840985 & 6.0193780131 \\
\hline & & 0.10 & 186.7358687239 & 4.4410088793 & 255.0797494461 & 4.4520489607 \\
\hline & & 0.30 & 64.3155063451 & 2.0244287200 & 89.2020341712 & 2.0259651623 \\
\hline & & 0.50 & 1.5604846313 & 2.0244287200 & 40.7569375637 & 1.5611422914 \\
\hline & \multirow[t]{8}{*}{0.2} & 0.00 & 370.0000576120 & 370.0000477061 & 500.0000655915 & 500.0000331334 \\
\hline & & 0.01 & 341.8375030358 & 38.4578607779 & 462.2452526816 & 39.5013005888 \\
\hline & & 0.03 & 293.0596802356 & 14.2482021597 & 396.7846634952 & 14.3827974754 \\
\hline & & 0.05 & 252.6450009210 & 8.9792734190 & 342.4723183628 & 9.0302521363 \\
\hline & & 0.07 & 218.9544992262 & 6.6755150638 & 297.1358039796 & 6.7024702255 \\
\hline & & 0.10 & 178.2991377127 & 4.9313310869 & 242.3400934144 & 4.9451380394 \\
\hline & & 0.30 & 57.2166373165 & 2.2098772863 & 78.2865567375 & 2.2118022389 \\
\hline & & 0.50 & 24.6972750753 & 1.6794079501 & 33.8062753712 & 1.6802371690 \\
\hline
\end{tabular}
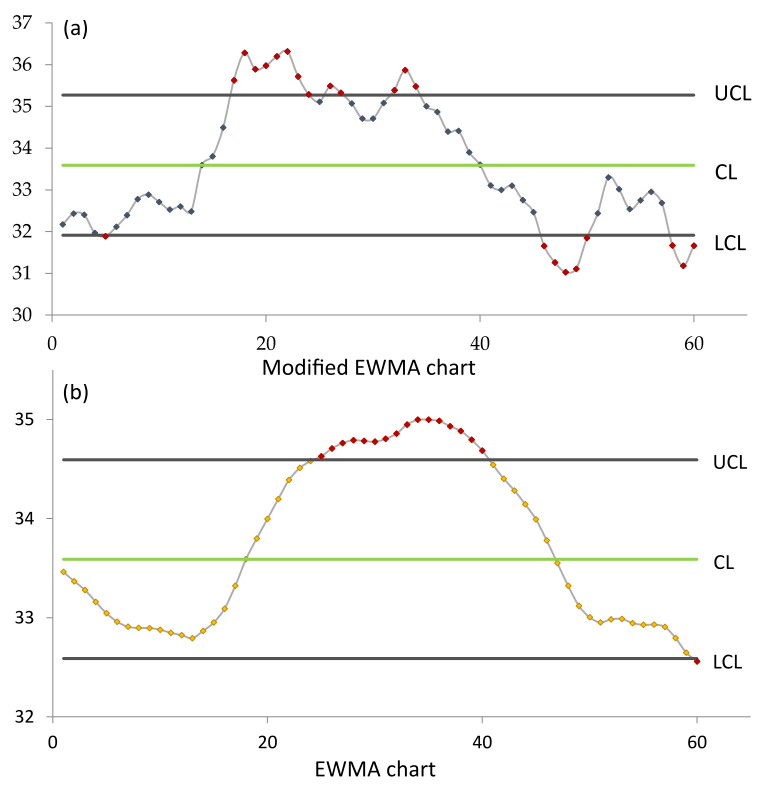

Fig. 2 The detection of the process with real data: (a) the modified EWMA and (b) the EWMA.

ing. The exponential smoothing parameter 0.1 is recommended. In addition, the performances of the modified and standard EWMA control charts were compared using real-world exchange rates data, the result indicating that the modified EWMA control chart was more efficient than the standard one.

Acknowledgements: This research was funded by King Mongkut's University of Technology North Bangkok and Thailand Science Research and Innovation (TSRI) Contract No. RSA 6280086.

\section{REFERENCES}

1. Montgomery DC (2009) Introduction to Statistical Quality Control, 6th edn, Wiley, New York.

2. Page ES (1954) Continuous inspection schemes. Biometrika 41, 100-115.

3. Roberts SW (1959) Control chart tests based on geometric moving average. Technometrics 1, 239-250.

4. Yashchin M (1993) Performance of CUSUM control schemes for serially correlated observations. Technometrics 35, 37-52.

5. Zhang NF (1998) A statistical control chart for stationary process data. Technometrics 40, 24-38.

6. Psarakis S, Papaleonida GEA (2007) SPC procedures for monitoring autocorrelated processes. Qual Technol Quant M 4, 501-540. 
Table 5 Comparison of two-sided of ARL for MA(1) on EWMA and modified EWMA charts using explicit formulas when $\mu=2$ and $c=50 \lambda$.

\begin{tabular}{|c|c|c|c|c|c|c|}
\hline \multirow{2}{*}{$\lambda$} & \multirow[t]{2}{*}{$\theta$} & \multirow{2}{*}{$\begin{array}{c}\text { Shift size } \\
(\delta)\end{array}$} & \multicolumn{2}{|c|}{$\mathrm{ARL}_{0}=370$} & \multicolumn{2}{|c|}{$\mathrm{ARL}_{0}=500$} \\
\hline & & & EWMA & Modified & EWMA & Modified \\
\hline \multirow[t]{16}{*}{0.05} & -0.4 & 0.00 & 370.0000555149 & 370.0000795892 & 500.0000440249 & 500.0000713397 \\
\hline & & 0.01 & 317.1741116242 & 41.9969256683 & 428.5634950788 & 43.25458952521 \\
\hline & & 0.03 & 235.1585236152 & 15.5352072476 & 317.6537185179 & 15.6990475039 \\
\hline & & 0.05 & 176.3481718462 & 9.7284228105 & 238.1243548315 & 9.7904827888 \\
\hline & & 0.07 & 133.6885773383 & 7.1854323906 & 180.4356556364 & 7.2182005835 \\
\hline & & 0.10 & 89.9632614742 & 5.2597771485 & 121.3057315405 & 5.2765129737 \\
\hline & & 0.30 & 10.7691191351 & 2.2829992334 & 14.2109276294 & 2.2686198289 \\
\hline & & 0.50 & 2.8940179432 & 1.69237742929 & 3.5613189677 & 1.6933433542 \\
\hline & 0.4 & 0.00 & 370.0000314578 & 370.0000810411 & 500.0000652911 & 500.0000523870 \\
\hline & & 0.01 & 314.6794551262 & 45.7317530918 & 425.1899218557 & 47.2269890336 \\
\hline & & 0.03 & 229.7650783509 & 17.0276497843 & 310.3599859730 & 17.2247307716 \\
\hline & & 0.05 & 169.7934005538 & 10.6713120635 & 229.2601018536 & 10.7461007259 \\
\hline & & 0.07 & 126.9222352329 & 7.8801356635 & 171.2852755031 & 7.9196410929 \\
\hline & & 0.10 & 83.7224337918 & 5.7625654284 & 112.8660125465 & 5.7827413520 \\
\hline & & 0.30 & 9.1221667504 & 2.4553179300 & 11.9836764191 & 2.4580722709 \\
\hline & & 0.50 & 2.4506522991 & 1.8128724325 & 2.9617326913 & 1.8140418723 \\
\hline \multirow[t]{16}{*}{0.10} & -0.4 & 0.00 & 370.0000518336 & 370.0000614837 & 500.0000776587 & 500.0000873733 \\
\hline & & 0.01 & 352.1496772110 & 35.1675420174 & 480.5671128914 & 36.0364577678 \\
\hline & & 0.03 & 319.9954347429 & 12.9784424848 & 445.5615061301 & 13.0894176155 \\
\hline & & 0.05 & 291.9356456494 & 8.1870306923 & 415.0336802266 & 8.2290068857 \\
\hline & & 0.07 & 267.3327564446 & 6.0967379490 & 388.3095198819 & 6.1189301589 \\
\hline & & 0.10 & 235.7921446525 & 4.5165264412 & 354.1704037426 & 4.5278976817 \\
\hline & & 0.30 & 119.5488019007 & 2.0594906242 & 234.0545686064 & 2.0610780140 \\
\hline & & 0.50 & 73.0760688952 & 1.5851215979 & 202.31849758374 & 1.5858037164 \\
\hline & 0.4 & 0.00 & 370.0000434468 & 370.0000381773 & 500.0000679211 & 500.0000665742 \\
\hline & & 0.01 & 345.4281185687 & 36.7693566544 & 467.64025996536 & 37.7207921966 \\
\hline & & 0.03 & 302.2029432208 & 13.5999906834 & 410.5608960138 & 13.7220985121 \\
\hline & & 0.05 & 265.6509248565 & 8.5776745459 & 362.1207155824 & 9.0302521363 \\
\hline & & 0.07 & 234.5756240775 & 6.3841434405 & 320.7953553002 & 6.4085834870 \\
\hline & & 0.10 & 196.1945491439 & 4.7245233411 & 269.5427528719 & 4.7370462384 \\
\hline & & 0.30 & 72.6935285465 & 2.1383209265 & 102.2625833543 & 2.1400704197 \\
\hline & & 0.50 & 34.6069696123 & 1.6357556044 & 49.3435046695 & 1.6365094477 \\
\hline
\end{tabular}

Table 6 Comparison of two-sided of ARL for MA(1) on EWMA and modified EWMA charts using explicit formulas when $\mathrm{ARL}_{0}=370, \mu=33.567, \theta=-0.869$, and $\beta_{0}=0.8207$.

\begin{tabular}{|c|c|c|c|c|}
\hline \multirow{2}{*}{$\begin{array}{l}\text { Shift size } \\
(\delta)\end{array}$} & \multicolumn{2}{|c|}{$\lambda=0.05$} & \multicolumn{2}{|c|}{$\lambda=0.10$} \\
\hline & $\begin{array}{c}\text { EWMA } \\
h=1.738 \times 10^{-11}\end{array}$ & $\begin{array}{c}\text { Modified } \\
b=1.976 \times 10^{-14}\end{array}$ & $\begin{array}{c}\text { EWMA } \\
h=2.3226 \times 10^{-14}\end{array}$ & $\begin{array}{c}\text { Modified } \\
b=2.07 \times 10^{-15}\end{array}$ \\
\hline 0.00 & 370.1606511895 & 370.9704083281 & 370.1086602485 & 370.0950486051 \\
\hline 0.01 & 262.9495082304 & 240.8173740827 & 240.8173740827 & 219.2229808369 \\
\hline 0.03 & 136.0663459955 & 96.3929255627 & 105.3513596562 & 87.9774945611 \\
\hline 0.05 & 72.7559093150 & 41.8842059927 & 48.1559932900 & 35.3549720373 \\
\hline 0.07 & 40.1994636558 & 19.0826033618 & 23.0674462751 & 15.8581662799 \\
\hline 0.10 & 17.6114490930 & 6.7114526651 & 8.5069999491 & 5.2864805809 \\
\hline 0.30 & 1.1720315932 & 1.0123817372 & 1.0243050587 & 1.0065595855 \\
\hline 0.50 & 1.0069206108 & 1.0001673887 & 1.0004345640 & 1.0000703639 \\
\hline
\end{tabular}

7. Prajapati DR (2015) Effectiveness of conventional CUSUM control chart for correlated observations. Int J Model Optim 5, 135-139.

8. Mawonike R, Nkomo V (2015) Univariate statistical process control of super saver beans: A case of RMV supermarket, Zimbabwe. J Manag Sci 5, 48-58.

9. Patel AK, Divecha J (2011) Modified exponentially weighted moving average (EWMA) control chart for an analytical process data. J Chem Eng Mater Sci 2, 12-20.

10. Khan N, Aslam M, Jun C (2017) Design of a control chart using a modified EWMA statistic. Qual Reliab Engng Int 33, 1095-1104.

11. Mastrangelo CM, Montgomery DC (1995) SPC with correlated observations for the chemical and process industried. Qual Reliab Eng Int 11, 79-89.

12. VanBrackle L, Reynolds MR (1997) EWMA and CUSUM control charts in the presence of correlation. Commun Stat-Simul C 26, 979-1008.

13. Fu JC, Spiring FA, Xie H (2002) On the average run length of quality control schemes using a markov chain approach. Statist Probab Lett 56, 369-380.

14. Zhang J, Li Z, Chen B, Wang Z (2014) A new exponentially weighted moving average control chart for monitoring the coefficient of variation. Comput Ind Eng 78, 205-212.

15. Sukparungsee S, Areepong Y (2009) A study of the performance of EWMA chart with transformed 
Weibull observations. Thail Stat 7, 179-191.

16. Busaba J, Sukparungsee S, Areepong Y, Mititelu G (2012) Numerical approximations of average run length for AR(1) on exponential CUSUM. In: Proceeding of International Multi Conference of Engineers and Computer Scientists, Hong Kong.

17. Suriyakat W, Areepong Y, Sukparungsee S, Mititelu G (2012) On EWMA procedure for an AR(1) observations with exponential white noise. Int J Pure Appl Math 77, 73-83.

18. Areepong Y (2019) Explicit formulas of average run length for a moving average control chart monitoring the number of defective products. IJPAM 80, 331-343.

19. Petcharat K, Areepong Y, Sukparungsee S (2013) Exact solution of average run length of EWMA chart for MA(q) processes. Far East J Math Sci 78, 291-300.

20. Petcharat K, Sukparungsee S, Areepong Y (2015) Exact solution of average run length for the cumulative sum chart for a moving average process of order q. ScienceAsia 41, 141-147.

21. Phantu S, Sukparungsee S, Areepong Y (2016) Explicit expressions of average run length of moving average control chart for Poisson integer valued autoregressive model. In: Proceeding of International Multi Conference of Engineers and Computer Scientists, Hong Kong.
22. Peerajit W, Areepong Y, Sukparungsee S (2019) Explicit analytical solutions of ARL of CUSUM chart for a long-memory SARFIMA model. Commun StatSimul C 48, 1176-1190.

23. Sunthornwat R, Areepong Y, Sukparungsee S, Mititelu G (2017) Average run length of the longmemory autoregressive fractionally integrated moving average process of the exponential weighted moving average control chart. Cogent Math Stat 4, ID 1358536.

24. Vargas VC, Lopes LF, Souza AM (2004) Comparative study of the performance of the CUSUM and EWMA control charts. Comput Ind Eng 46, 707-724.

25. Areepong Y (2009) An integral equation approach for analysis of control charts. PhD thesis, Univ of Technology, Australia.

26. Suriyakat W, Areepong Y, Sukparungsee S, Mititelu G (2012) Analytical method of average run length for trend exponential AR(1) process in EWMA procedure. Int J Appl Math 42, 250-253.

27. Phanyaem S, Areepong Y, Sukparungsee S (2014) Numerical integral of average run length of CUSUM control chart for ARMA process. Int J Appl Phys Math 4, 232-235.

28. Sofonea M, Han W, Shillor M (2005) Analysis and Approximation of Contact Problems with Adhesion or Damage, Chapman \& Hall/CRC, New York. 